\title{
TREM2 upregulation correlates with 5-hydroxymethycytosine enrichment in Alzheimer's disease hippocampus
}

\author{
Naiara Celarain ${ }^{1}$, Javier Sánchez-Ruiz de Gordoa ${ }^{1,2,5}$, María Victoria Zelaya ${ }^{3}$, Miren Roldán ${ }^{1}$, Rosa Larumbe ${ }^{1,2}$, \\ Laura Pulido ${ }^{1,2,5}$, Carmen Echavarri ${ }^{1,4}$ and Maite Mendioroz ${ }^{1,2^{*}}$
}

\begin{abstract}
Background: Recent genome-wide association studies revealed TREM2 rs75932628-T variant to be associated with Alzheimer's disease (AD) and other neurodegenerative diseases. However, the role that TREM2 plays in sporadic AD is largely unknown. Our aim was to assess messenger RNA (mRNA) expression levels and DNA methylation profiling of TREM2 in human hippocampus in AD brain. We measured TREM2 mRNA levels in the hippocampus in a cohort of neuropathologically confirmed controls and pure AD cases showing no other protein deposits than $\beta$-amyloid and phosphorylated tau. We also examined DNA methylation levels in the TREM2 transcription start site (TSS)-associated region by bisulfite cloning sequencing and further extended the study by measuring 5 -hydroxymethycytosine (5hmC) enrichment at different regions of TREM2 by $5 \mathrm{hmC}$ DNA immunoprecipitation combined with real-time GPCR.
\end{abstract}

Results: A 3.4-fold increase in TREM2 mRNA levels was observed in the hippocampus of AD cases compared to controls ( $p=1.1 \mathrm{E}-05)$. Interestingly, TREM2 methylation was higher in AD cases compared to controls $(76.2 \% \pm 15.5$ versus $57.9 \% \pm 17.1 ; p=0.0016)$. Moreover, TREM2 mRNA levels in the AD hippocampus correlated with enrichment in $5 \mathrm{hmC}$ at the TREM2 gene body $(r=0.771 ; p=0.005)$.

Conclusions: TREM2 mRNA levels are increased in the human hippocampus in AD cases compared to controls. DNA methylation, and particularly 5hmC, may be involved in regulating TREM2 mRNA expression in the AD brain. Further studies are guaranteed to investigate in depth the role of $5 \mathrm{hmC}$ in $\mathrm{AD}$ and other neurodegenerative disorders.

Keywords: Gene expression, Alzheimer's disease, Epigenetics, DNA methylation, TREM2, 5-Hydroxymethycytosine

\section{Background}

The triggering receptor expressed on myeloid cells 2 (TREM2) gene [EMBL: AB601768] encodes a transmembrane glycoprotein receptor that activates the innate immune response in macrophages and dendritic cells. In the brain, TREM2 is expressed in microglia and it seems to promote phagocytosis of apoptotic neurons, cellular debris, and misfolded proteins by recognizing specific endogenous ligands on the surface of apoptotic cells [13]. At the same time, TREM 2 retards the inflammatory response by repressing microglial cytokine production

\footnotetext{
* Correspondence: maitemendilab@gmail.com

${ }^{1}$ Neuroepigenetics Laboratory, Navarrabiomed-Navarra Institute for Health Research (IdiSNA), c/ Irunlarrea, Pamplona, Navarra 31008, Spain

2Department of Neurology, Complejo Hospitalario de Navarra, Pamplona, Navarra 31008, Spain

Full list of author information is available at the end of the article
}

[3]. Thus, TREM2 seems critical to maintain brain homeostasis in response to tissue damage.

Lately, genome-wide association studies (GWAS) revealed TREM2 gene variant rs75932628-T to be associated with Alzheimer's disease (AD) and other neurodegenerative diseases, such as Parkinson's disease, frontotemporal dementia and amyotrophic lateral sclerosis [4-7]. However, the mechanisms by which TREM2 mutations might increase the risk of $A D$ remain elusive. A recent study showed that loss of a single copy of TREM2 significantly altered the morphological phenotype of $\beta$-amyloid plaque-associated microglia in the APPPS1-21 AD mouse model [8]. In the case of rs75932628-T variant, arginine to histidine substitution $(\mathrm{R} 47 \mathrm{H})$ may have a significant effect on the ligand binding affinity and reduce the phagocytic activity [9-12]. 
Specifically, TREM2 is supposed to promote phagocytosis of $A \beta 42$ peptides, preventing $\beta$-amyloid accumulation and downstream neurotoxic effects [13, 14]. Most recently, it was shown that $\mathrm{R} 47 \mathrm{H}$ impairs detection of lipid ligands known to associate with fibrillar $\beta$-amyloid [15]. Therefore, impairment in clearance of $A \beta 42$ and cellular debris may in part explain the increased risk of $\mathrm{AD}$ in carriers of TREM2 gene variants [16].

On the other hand, the role of non-mutated TREM2 in sporadic $\mathrm{AD}$ also needs further investigations. Notably, TREM2 messenger RNA (mRNA) was upregulated in amyloid plaque-associated versus plaque-free brain tissue of aged APP23 mice, a transgenic AD mouse model [17]. Using another transgenic mouse model, TREM2 was found to be overexpressed in microglia during disease progression [14]. TREM2 expression has also been assessed in humans. According to a microarraybased expression study on postmortem brain samples from normal individuals, highest levels of TREM2 mRNA were identified in the lobar white matter, substantia nigra, and medulla [18]. However, studies on TREM2 expression in the $\mathrm{AD}$ human brain are scarce and controversial with some authors showing increased levels of TREM2 in AD [19-21], while others reported downregulation of TREM2 in the AD context [22].

Here, we investigated mRNA levels of TREM2 in the human hippocampus in a cohort of neuropathologically defined "pure" AD cases and controls. Moreover, to assess epigenetic mechanisms potentially involved in regulating TREM2 in $\mathrm{AD}$, we profiled DNA methylation at different regulatory regions of the TREM2 gene in the AD hippocampus.

\section{Results}

TREM2 mRNA levels are upregulated in Alzheimer's disease hippocampus

We first measured TREM2 mRNA levels in hippocampal samples from Alzheimer's disease (AD) cases and controls by RT-qPCR. Four samples did not pass the RNA quality threshold so were not included in the experiments (see TREM2 mRNA expression analysis in the "Methods" section). Eventually, $26 \mathrm{AD}$ cases were compared to 12 controls. None of the subjects included in the study was carrying the TREM2 rs75932628-T variant, in accordance with the low frequency of the variant allele in the European ancestry population [7]. A 3.4-fold increase in TREM2 mRNA levels was observed in the hippocampus of $\mathrm{AD}$ cases compared to controls (mean \pm SD mRNA levels in $A D$ versus controls: $6.65 \pm 4.30 \%$ versus $1.73 \pm 1.24 \% ; p=1.1 \mathrm{E}-05$ ) (Fig. 1a). Next, a disease-staging analysis was conducted to investigate changes of TREM 2 mRNA levels considering AD severity. We found that TREM2 mRNA levels significantly increased across AD stages ( $p=0.003$; Fig. 1b). GamesHowell post hoc analysis showed that TREM2 mRNA expression was significantly different between control and Braak stages III-IV $(p=0.003)$ and between control and Braak stages V-VI $(p=0.035)$ (Fig. 1b).

Then, a logistic regression model was designed to identify adjusted estimates of the association of TREM2 mRNA levels with $A D$ status (control $=0 ; A D=1$ ). Age and gender were included into the model in order to adjust for potentially confounding variables, since there were significant age differences between the control and $\mathrm{AD}$ group and a statistical trend was found towards higher proportion of male in the control group (see Human brain samples and neuropathological examination in the "Methods" section). As shown in Table 1, TREM2 mRNA expression remained as an independent predictor of $\mathrm{AD}$ status after adjusting for age and gender $(p=0.026)$ (Table 1).

We further tested if differences in TREM2 mRNA levels resulted from an increase in a particular type of TREM2 splice variant. Two different types of TREM2 transcripts were identified in the Reference Sequence (RefSeq) database, i.e., TREM2 transcript variant 1
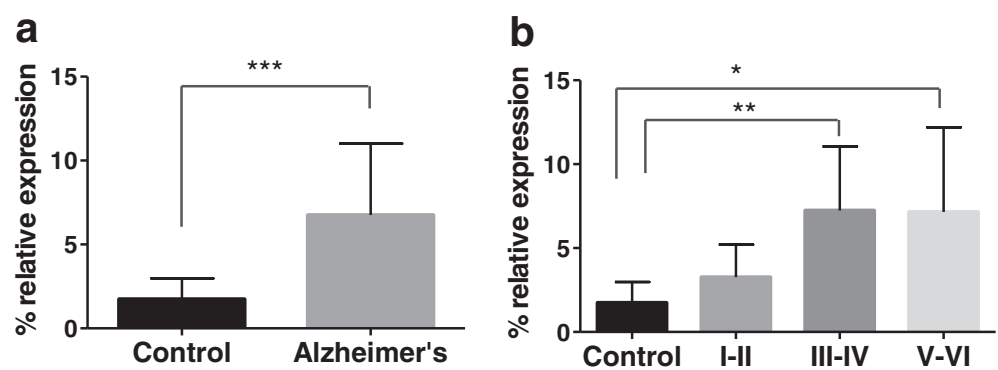

Fig. 1 TREM2 mRNA expression is increased in human hippocampus in Alzheimer's disease (AD). a The graph shows a significant 3.4-fold increase in TREM2 mRNA levels in AD hippocampal samples compared to control hippocampal samples. b TREM2 mRNA expression increases across AD stages, as shown when TREM2 mRNA expression levels are sorted by Braak \& Braak stages. Bars represent percentage of TREM2 mRNA expression relative to the geometric mean of HPRT and ACTB housekeeping genes expression. Vertical lines represent the standard error of the mean. ${ }^{*} p$ value $<0.05 ;{ }^{* *} p$ value $<0.005 ;{ }^{* * *} p$ value $<0.0005$ 
Table 1 Adjusted logistic regression model to predict AD status

\begin{tabular}{lllllll}
\hline Variable & $\mathrm{B}$ & Wald & $p$ value & OR & $95 \% \mathrm{Cl}$ inferior & $95 \% \mathrm{Cl}$ superior \\
\hline TREM2 mRNA levels & 0.709 & 4.964 & $0.026^{*}$ & 2.031 & 1.089 & 3.789 \\
Gender (female) & 0.369 & 0.108 & 0.742 & 1.446 & 0.161 & 13.015 \\
Age $<65$ yo & 1.737 & 2.383 & 0.123 & 0.176 & 0.019 & 1.597 \\
Constant & 1.035 & 0.572 & 0.45 & 0.355 & - & - \\
\hline
\end{tabular}

Alzheimer status (control $=0 ; A D=1$ ) was considered as the dependent variable, and TREM2 mRNA expression levels, gender, and age were included as covariates. $B$ regression coefficient, $O R$ odds ratio, yo years old, ${ }^{*} p$ value $<0.05$

(NM_018965) and TREM2 transcript variant 2 (NM_001271821) (Additional file 1: Figure S1.A). Next, two sets of primers that separately amplify each TREM2 splice variant were designed (Additional file 1: Table S1 and Figure S1.A). Interestingly, we found that mRNA levels of each of both transcripts were significantly higher in the hippocampus from AD patients compared to controls (Additional file 1: Figure S1.B). Nonetheless, the most prominent splice variant in the hippocampal tissue was TREM2 transcript variant 1 (NM_018965), suggesting that the majority of mRNA changes may be accounted for by an increase in that particular splice variant.

\section{TREM2 mRNA levels positively correlate with $\beta$-amyloid and $\mathrm{p}$-tau burden}

Next, TREM2 mRNA expression was examined in more detail according to the neuropathological AD-related changes measured and recorded from the hippocampus sections. The amyloid plaque score (APS) and average area of both $\beta$-amyloid deposition and hyperphosphorylated tau ( $\mathrm{p}$-tau) were quantitatively measured as described in the "Methods" section by using ImageJ software [23] (Additional file 1: Figure S2) in the whole set of 30 $\mathrm{AD}$ cases and 12 controls included in the study. Interestingly, a significant association between APS and TREM2 mRNA levels was found in the human hippocampus $(r=$ $0.593, p=1.4 \mathrm{E}-04)$. In addition, the average area of $\beta$ amyloid burden in the hippocampus was also correlated with TREM2 mRNA levels ( $r=0.602, p=1.0 \mathrm{E}-04)$, suggesting that TREM2 mRNA expression parallels the molecular changes induced by $\beta$-amyloid deposition. Since we had found an increased TREM2 mRNA expression across Braak stages, a positive correlation with p-tau deposits was expected. Indeed, the average area of p-tau deposition, which included neurofibrillary tangles, neuropil threads, and neuritic plaques, was positively correlated to TREM2 mRNA levels $(r=0.509, p=0.002)$.

\section{DNA methylation in the TREM2 transciption start site (TSS)-associated region is increased in AD cases compared to controls}

To further explore if differences in TREM2 mRNA levels between $\mathrm{AD}$ cases and controls were related to DNA methylation changes, bisulfite cloning sequencing was conducted in a subset of six controls and 10 Alzheimer's hippocampal samples. Bisulfite cloning sequencing assesses changes in DNA methylation which includes modifications in 5-methylcytosine $(5 \mathrm{meC})$ and 5-hydroxymethylcytosine $(5 \mathrm{hmC})$. There were no significant differences between control and $\mathrm{AD}$ groups regarding age (52 \pm 27.9 years versus $74.8 \pm 13.7 ; p$ value $=0.107$ ) or gender (male percentage) (62.5 versus $62.5 \%$; $p$ value $=1.0)$. Moreover, PMI was similar in both groups $(7.1 \pm 2.9 \mathrm{~h}$ versus $7.6 \pm 5.6 \mathrm{~h} ; p$ value $=0.821)$.

Percentage of DNA methylation at seven individual cytosine guanine dinucleotides (CpGs) in the TSS-associated region of TREM2 gene (Fig. 2a) was measured by sequencing a minimum of 20 clones per individual. Then, average DNA methylation for the entire amplicon was calculated in each individual. Following that approach, a significant increase in DNA methylation at the TSS-associated region of TREM2 gene was observed in the AD cases group compared to the control group $(76.2 \pm 15.5 \%$ versus $57.9 \pm$ $17.1 \% ; p=0.0016$ ) (Fig. 2b). All seven interrogated CpGs at the TSS-associated region showed a significant $(p<0.05)$ gain in DNA methylation in AD cases compared to controls (Table 2). After Bonferroni correction, differences between $\mathrm{AD}$ and controls in the average DNA methylation for the whole amplicon and DNA methylation in the first $\mathrm{CpG}$ of the amplicon ( $\mathrm{CpG}$ at position 24) remained significant (adjusted $p$ value $=0.00625$; Table 2). Interestingly, this result did not follow the classical model of epigenetic regulation where gain in methylation in the promoter region correlates with a decrease in gene expression. To improve the specificity of our assay, we used a negative control by bisulfite sequencing an amplicon located at -874 bp relative to TREM2 transcription start site (Additional file 1: Figure S3). There were no statistically significant differences between AD and control group (90.7 \pm 3.9 versus $92.5 \pm 6.45 ; p$ value $=0.664$ ) for this particular region. We also wanted to test the relationship between average DNA methylation and mRNA expression for the TREM2 gene. No correlation was found between DNA methylation percentage measured by bisulfite sequencing and TREM2 mRNA levels in our set of samples $(r=0.391$, $p$ value $=0.134)$. 


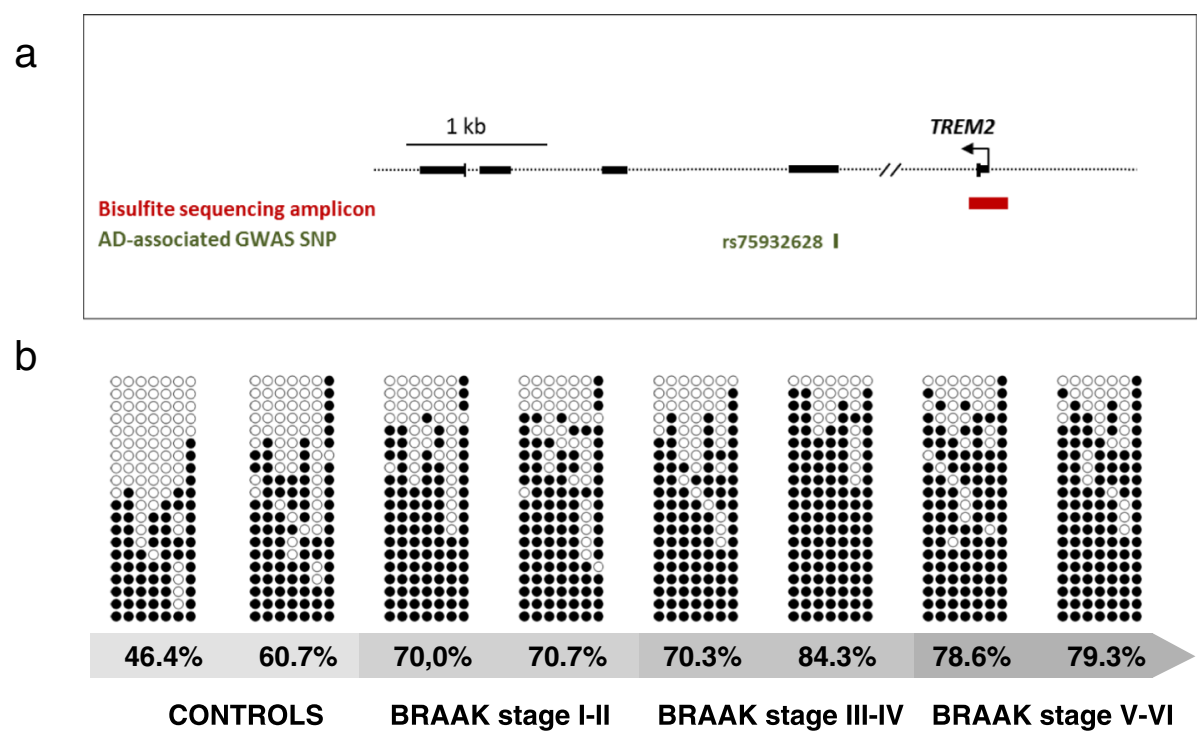

Fig. 2 DNA methylation levels in the TREM2 TSS-associated region in Alzheimer's disease (AD) and control hippocampus. a The figure shows the genomic position of the amplicon surveyed by bisulfite cloning sequencing at the TSS-associated region of the TREM2 gene. At the bottom of the figure, the GWAS-associated TREM2 variant (rs75932628) position is also shown. b The picture shows the methylation pattern at CpG site resolution of TREM2

TSS-associated region for different samples across AD progression. It is only an example of the bisulfite results for each group. Therefore, two individual subjects (samples) have been included per group. Black and white circles represent methylated and unmethylated cytosines, respectively. Each column symbolizes a unique CpG site in the examined amplicon, and each line represents an individual DNA clone. Global percentage of methylation for each analyzed sample (control or patient) at this particular amplicon is indicated at the bottom of each sample. Meth Amp methylation amplicon, GWAS genome-wide association studies

\section{$5 \mathrm{hmC}$ enrichment in the TREM2 gene body correlates with TREM2 mRNA levels in the AD hippocampus}

Next, we asked whether DNA methylation differences observed in $\mathrm{AD}$ cases were indeed the result of $5 \mathrm{hmC}$ changes, as higher $5 \mathrm{hmC}$ levels at specific sites have been related to increased gene expression [24, 25]. Since bisulfite cloning sequencing is unable to distinguish between $5 \mathrm{mC}$ and $5 \mathrm{hmC}$, we performed 5-hydroymethylated DNA immunoprecipitation (5hMeDIP) to selectively enrich DNA fragments containing $5 \mathrm{hmC}$, followed by quantification by RT-qPCR, in a group of $12 \mathrm{AD}$ cases and 5 controls. Following that procedure, we were able to estimate $5 \mathrm{hmC}$ levels not only at the TSS-associated region but also at exon 2 and at the 3 ' end of the gene. Noticeably, amplicon covering exon 2 included the rs75932628-T variant associated with AD in GWAS (Fig. 3a). No statistically significant differences in $5 \mathrm{hmC}$ enrichment were found between groups at TREM2 TSS-associated region (IP/INPUT fold change enrichment \pm SD in $\mathrm{AD}$ versus controls: $3.52 \pm 5.48$ versus $1.69 \pm 0.95 ; p$ value $=0.130)$, exon 2 (AD versus controls: $18.70 \pm 12.73$ versus $16.73 \pm 5.14 ; p$ value $=0.383)$ and $3^{\prime}$ end (AD versus controls: $9.76 \pm 7.72$ versus $5.77 \pm 2.91$; $p$ value $=0.633)$ (Fig. $3 \mathrm{~b})$.

Interestingly, a significant positive correlation was found between $5 \mathrm{hmC}$ enrichment in exon 2 and TREM2 mRNA levels $(r=0.669 ; p=0.005)$ (Fig. 3c) for the whole set of samples. When considering only the AD hippocampal samples, correlation between $5 \mathrm{hmC}$ enrichment in exon 2 and TREM2 mRNA expression was found to be even stronger $(r=0.771 ; p=0.005)$ (Fig. $3 \mathrm{~d})$.

Table 2 Averaged methylation of each CpG site in control and AD groups

\begin{tabular}{llllllllll}
\hline CpG position & 24 & 65 & 129 & 175 & 236 & 269 & 471 & Total & SD \\
\hline MR control group. \% & 60.4 & 68.10 & 47.9 & 50.00 & 62.5 & 31.2 & 85.4 & 57.91 & 17.1 \\
MR AD group. \% & 83.7 & 85.4 & 66.7 & 70.7 & 80.5 & 49.2 & 96.7 & 76.16 & 15.5 \\
Gain in methylation. \% & 23.3 & 17.3 & 18.8 & 20.7 & 18.00 & 18.00 & 11.3 & 18.25 & - \\
$p$ value & $0.0015^{*}$ & 0.0163 & 0.0353 & 0.0130 & 0.0180 & 0.0400 & 0.0121 & $0.0016^{*}$ & - \\
\hline
\end{tabular}

CpG position denotes the position of the dinucleotide CpG within the amplicon. Total denotes the averaged methylation percentage for the whole amplicon. MR methylation ratio, $A D$ Alzheimer's disease, $S D$ standard deviation. ${ }^{*}$ significant after Bonferroni correction (adjusted $p$ value $=0.00625$ ) 


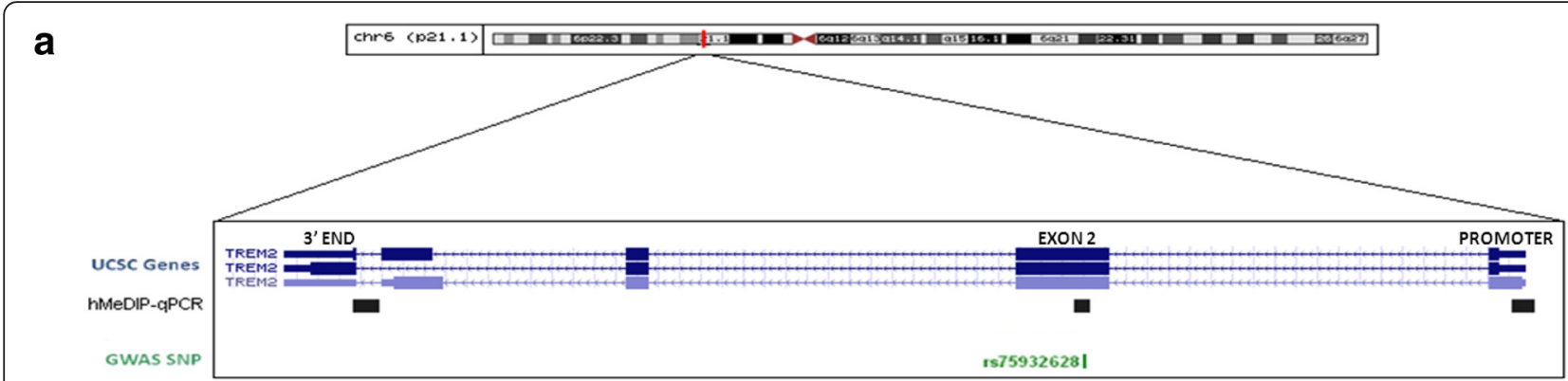

b
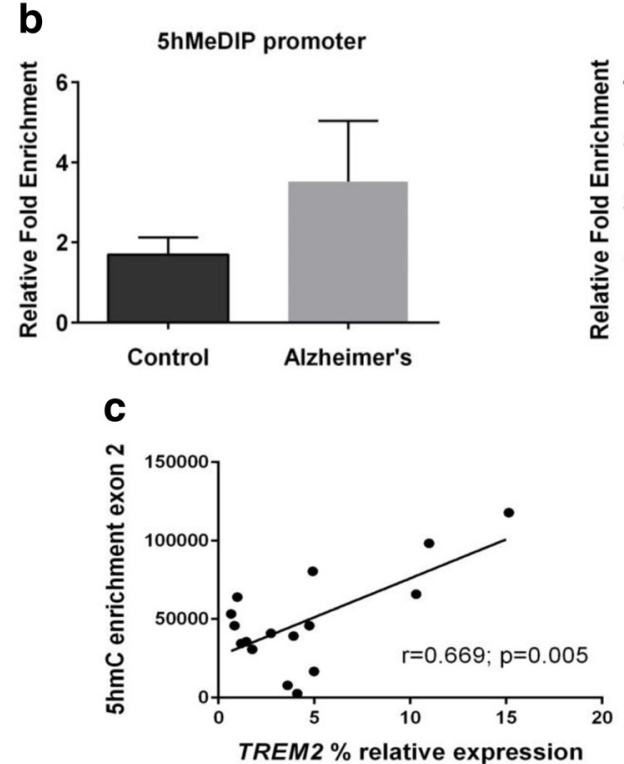
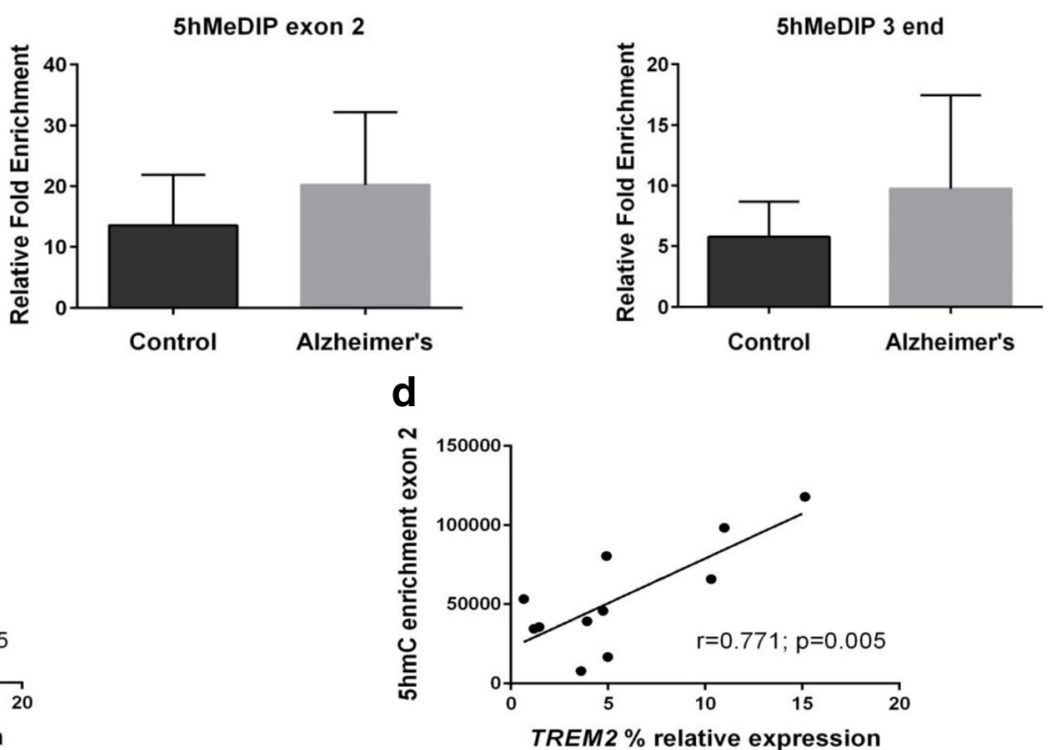

Fig. 3 5-Hydroxymethylated DNA profiling in Alzheimer's versus control hippocampus. a The figure shows the genomic locations of the amplicons (black bars) designed to assess the $5 \mathrm{hmC}$ enrichment at different regions of TREM2 by 5-hydroxymethylated DNA immunoprecipitation combined with RT-qPCR. UCSC Genes line denotes TREM2 gene predictions from the USCS track at the UCSC Genome Browser. hMeDIP-qPCR line shows the examined amplicons at the 3' end of the gene (left), the gene body at exon 2 (middle), and the TSS-associated region (right). The GWAS SNP line shows the genomic position of the TREM2 variant (rs75932628) which is included in the amplicon at exon 2. b 5hMeDIP experiments, using an antibody against 5-hydroxymethylcytosine (5-hmC), revealed no differences in levels of 5-hydroymethylated DNA on the TSS-associated region (2.6-fold enrichment, $p=0.130$ ) (left), and the gene body in exon $2(1.5$-fold enrichment, $p=0.383$ ) (middle) and the 3 ' end (1.7-fold enrichment, $p=0.633$ ) (right) of TREM2 in the hippocampus samples of AD patients compared to controls. The graphs $\mathbf{c}$ and $\mathbf{d}$ show statistically significant positive correlation between $5 \mathrm{hmC}$ enrichment in the TREM2 exon2 region for the whole set of samples (c) and only for the AD-affected hippocampus (d)

\section{Discussion}

An important finding of this study is that TREM2 mRNA is significantly upregulated in the human hippocampus in Alzheimer's disease (AD) and correlates with AD-related neuropathological changes. Furthermore, we show that TREM2 mRNA levels in the human hippocampus positively correlates with 5 -hydroxymethylcytosine $(5 \mathrm{hmC})$ enrichment in the TREM2 gene body, suggesting that $5 \mathrm{hmC}$ may be involved in regulating TREM2 in the AD context.

Our results on TREM2 mRNA expression are in high concordance with previous reports conducted in postmortem human brain samples that had showed upregulation of TREM2 in the inferior temporal cortex in sporadic AD cases [19] and a trend for TREM2 to be increased in the hippocampus of $\mathrm{AD}$ cases [20].
Accordingly, we found an increase in TREM2 mRNA levels in the $\mathrm{AD}$ hippocampus that indeed did reach consistent statistical significance in our set of samples. Nevertheless, replication of our result in a wider and independent cohort would be of most interest to support this finding.

Higher levels of TREM2 mRNA might reflect the activation of microglial cells in the $\mathrm{AD}$-affected brain, a phenomenon well known to occur in neurodegenerative diseases [26]. In fact, one explanation for high TREM2 mRNA levels in AD cases would be either that microglia increases TREM2 mRNA expression as an attempt to maintain the homeostasis after brain damage or a distinct increase in the proportion of reactive microglia in $\mathrm{AD}$ hippocampus, or even a combination of both. 
Further assays to measure proportion of cell population in $\mathrm{AD}$ and control cases, as with fluorescence-activated cell sorting (FACS), would be most helpful to address this issue. Whatever the case, it is clear that neuroprotective mechanisms fail to provide enough protection, given the ominous $\mathrm{AD}$ clinical course. In the context of TREM2 mutations, phagocytic function of TREM2 would be impaired, compromising even more the repair potential of neuroprotective mechanisms. In agreement with the latter idea, rs75932628-T variant in TREM2 accelerates the $\mathrm{AD}$ clinical course reducing the age at onset [27] and significantly shortening the disease duration [26].

Interestingly, TREM2 mRNA levels were positively correlated with both $\beta$-amyloid and p-tau burden in our study, as it would be expected of a protective mechanism trying, but not achieving, to repair brain damage. This positive correlation is in line with previous research conducted in AD mouse models that revealed upregulation of TREM 2 mRNA in the amyloid plaque boundaries [17], and those performed on human brain samples that showed positive correlation between TREM2 mRNA levels and p-tau burden in mid-temporal gyrus from $\mathrm{AD}$ patients [21]. Taken together, these data suggest that TREM2 overexpression could be a crucial but insufficient attempt to repair the brain tissue, as others have also proposed $[9,26]$.

The putative relationship between TREM2 mRNA expression and microglia activation in AD does not preclude epigenetic involvement in regulating TREM2. Indeed, the methylation pattern observed for TREM2 in our set of samples could be part of the epigenetic signature of activated microglia in the AD brain. Actually, epigenetic modulation of microglia phenotypes has been described in other neurodegenerative diseases, such as Parkinson disease [28]. However, epigenetic mechanisms involved in microglia activation still need further research. A limitation of our study is that our bisulfite sequencing analysis was performed in whole brain tissue, which indeed constitutes a mixed cell population. Hence, our bisulfite analysis is not specific for microglial cells, and epigenetic results may be biased by this cell heterogeneity, as epigenetic regulation is usually cell-type specific.

An interesting aspect of our finding is that higher levels of methylation in the TREM2 TSS-associated region parallel higher levels of mRNA expression. Classically, methylation of $\mathrm{CpG}$ islands located in promoter regions leads to silencing of gene expression. However, this rule does not fully apply to non-CpG island promoters, as it would be the case of the TREM2 TSSassociated region, which lacks a CpG island (Fig. 2a). The role of DNA methylation in non-CpG island promoters is under investigation, and methylation of poor-
CpG promoters does not preclude their activity [29]. Importantly, a positive correlation between promoter methylation and gene expression has also been described in other neurodegenerative conditions, such as Huntington disease [30]. Furthermore, cumulative evidence from recent studies suggests that regulation of gene expression by DNA methylation may be much more complex than the classical model description [31], and new epigenetic marks have been described, such as $5 \mathrm{hmC}$, that seems to work differently from 5 -methylcytosine $(5 \mathrm{mC})$ in controlling gene expression.

The $5 \mathrm{hmC}$ content varies drastically among different mammalian tissues, with the highest levels being found in the brain [32-34]. Moreover, the amount of $5 \mathrm{hmC}$ globally increases during normal brain aging, particularly in the hippocampus $[35,36]$. Interestingly, global $5 \mathrm{hmC}$ levels are higher in human hippocampus and parahippocampal gyrus in AD subjects compared to controls [25]. Furthermore, in postmortem human samples, global $5 \mathrm{hmC}$ levels positively correlate with $\beta$-amyloid and tau loads, in the frontal and temporal gyrus [37]. Regarding gene expression, it has been described that $5 \mathrm{hmC}$ may play a dual role in regulating both transcriptional activation and repression [38]. In the brain tissue, $5 \mathrm{hmC}$ is enriched in the gene bodies of actively transcribed genes, where it is directly correlated with expression levels of the corresponding genes $[25,39]$. Consistent with the latter statement, we found in the AD hippocampus a significant and positive correlation between $5 \mathrm{hmC}$ enrichment in the TREM2 gene body at exon 2 and TREM2 mRNA levels. Noticeably, the amplified region within exon 2 harbors the rs75932628-T variant previously associated to AD by GWAS. So, a cisregulatory effect on $5 \mathrm{hmC}$ levels would be worthy to be examined in future studies.

\section{Conclusions}

In summary, this study provides evidence that TREM2 mRNA is upregulated in the human hippocampus affected by AD. Our findings also suggest that $5 \mathrm{hmC}$ may play a role in regulating TREM2 mRNA expression, a gene related to the $\mathrm{AD}$ pathogenesis. Further studies are guaranteed to investigate in depth the regulatory role of $5 \mathrm{hmC}$ in $\mathrm{AD}$ and other neurodegenerative disorders.

\section{Methods}

Human brain samples and neuropathological examination We conducted an observational, case-control study comparing postmortem hippocampal samples from $\mathrm{AD}$ patients and control hippocampus. Frozen postmortem hippocampal samples from 30 Alzheimer's disease (AD) cases and 12 controls were provided by the Navarrabiomed Brain Bank. After death, half brain specimens from donors were cryopreserved at $-80{ }^{\circ} \mathrm{C}$. 
Neuropathological examination was completed following the usual recommendations [40]. Assessment of $\beta$ amyloid deposit was carried out by immunohistochemical staining of paraffin-embedded sections (3- to $5-\mu \mathrm{m}$ thick) with a mouse monoclonal (S6F/3D) anti $\beta$ amyloid antibody (Leica Biosystems Newcastle Ltd, Newcastle upon Tyne, UK). Evaluation of neurofibrillary pathology was performed with a mouse monoclonal antibody anti-human PHF-TAU, clone AT-8, (Tau AT8) (Innogenetics, Gent, Belgium), which identifies hyperphosphorylated tau ( $\mathrm{p}$-tau) [41]. The reaction product was visualized using an automated slide immunostainer (Leica Bond Max) with Bond Polymer Refine Detection (Leica Biosystems Newcastle Ltd).

Subjects received diagnosis of pathophysiologically proved AD dementia according to the updated National Institute on Aging-Alzheimer's Association guidelines [42]. Subjects were further classified, based on neurofibrillary pathology, as follows: control $(n=12)$; Braak stages I-II $(n=3)$; Braak stages III-IV $(n=17)$; and Braak stages V-VI $(n=10)[41,43]$. Importantly, to avoid spurious associations, those individuals showing coincident protein deposits different from $\mathrm{p}$-tau or $\beta$ amyloid were not eligible for the study. This approach maximizes chances of finding true associations with $\mathrm{AD}$, even though reducing the final sample size. Neuropathological and demographic features of subjects, including age, gender, and postmortem interval are listed in Additional file 1: Table S2. There were significant age differences between the control and AD group $(50.7 \pm$ 21.5 years versus $82.3 \pm 11.3$ years, $p<0.01$ ), and a statistical trend was found towards higher proportion of male in the control group (66.7versus $34.5 \%, p=0.87$ ). The postmortem interval (PMI) was not significantly different between groups $(8.2 \pm 4.2 \mathrm{~h}$ in the control group versus $7.9 \pm 7.1 \mathrm{~h}$ in the AD group; $p=0.91$ ).

\section{Ethics, consent, and permissions}

The Ethics Committee of the "Complejo Hospitalario de Navarra" approved the use of human subjects for this study (Pyto 90/2014). Written informed consent was obtained from all subjects or next of kin.

\section{Genotyping of TREM2 variant rs75932628-T}

We wanted to assess whether any subject included in our study was harboring the TREM2 rare variant rs75932628-T. To that end, we genotyped the TREM2 variant rs75932628-T as it has been previously described [44]. In brief, a PCR reaction was carried out to amplify a 172 base pair (bp) amplicon spanning the rs75932628 variant. Next, a restriction fragment length polymorphism analysis was performed by using the restriction enzyme HhaI (Thermo Fisher Scientific Inc., Waltham, MA, USA). The change from $\mathrm{C}$ to $\mathrm{T}$ in rs75932628 results in loss of the HhaI restriction enzyme site. When separated by electrophoresis, digested PCR fragments from homozygous wild-type subjects show two bands of 89- and 84-bp length, whereas heterozygous subjects harboring TREM2 variant rs75932628-T display an additional 172 bp band, corresponding to the allele that has lost the restriction enzyme site.

\section{TREM2 mRNA expression analysis}

Total RNA was isolated from hippocampus homogenates using RNeasy Lipid Tissue Mini kit (QIAGEN, Redwood City, CA, USA), following manufacturer's instructions. Genomic DNA was removed with a recombinant DNase (TURBO DNA-free ${ }^{\mathrm{Tm}}$ Kit, Ambion, Inc., Austin, TX, USA). RNA integrity was checked by $1.25 \%$ agarose gel electrophoresis under denaturing conditions. Concentration and purity of RNA were both evaluated with NanoDrop spectrophotometer. Only those RNA samples showing $260 \mathrm{~nm} / 280 \mathrm{~nm}$ absorbance ratios between 1.8 and 2.2 and $260 \mathrm{~nm} / 230 \mathrm{~nm}$ absorbance ratios higher than 1.8 were considered high enough quality to be included in the study. Complementary DNA (cDNA) was reverse transcribed from $1500 \mathrm{ng}$ total RNA per sample with SuperScript ${ }^{\bullet}$ III First-Strand Synthesis Reverse Transcriptase (Invitrogen, Carlsbad, CA, USA) and primed with a mixture of oligo-d (T) and random primers.

Real-time qPCR (RT-qPCR) reactions were performed in triplicate for each sample using Power SYBR ${ }^{\oplus}$ Green PCR Master Mix (Invitrogen, Carlsbad, CA, USA) in a 7300 real-time PCR System (Applied Biosystems, Foster City, CA, USA). Experiments were repeated twice within independent cDNA sets. Sequences of primer pair were designed by using real-time PCR tool (IDT, Coralville, IA, USA) and are listed in Additional file 1: Table S1. The PCR amplicon sizes range from 116 to $184 \mathrm{bp}$. All the primers used in RT-qPCR assays were tested for specificity. During designing the primer sets, a Basic Local Alignment Search Tool (BLAST) versus entire mRNA RefSeq database was performed to verify the correct and unique alignment of the fragment. Moreover, dissociation curves were obtained for each assay to check that curves show a single peak with no shoulder. After amplification, we also checked that RT-qPCR reaction had generated a single amplicon of the correct size by performing agarose gel electrophoresis.

The thermal cycling conditions consisted of an initial denaturation step at $95{ }^{\circ} \mathrm{C}$ for $10 \mathrm{~min}$ followed by $40 \mathrm{cy}$ cles of $15 \mathrm{~s}$ at $95{ }^{\circ} \mathrm{C}$ and $1 \mathrm{~min}$ at $60{ }^{\circ} \mathrm{C}$. The geometric mean of $A C T B$ and HPRT housekeeping genes was used to normalize TREM2 mRNA levels [45], and nontemplate reactions were included as negative controls in each run. Relative expression level of TREM2 mRNA in 
a particular sample was calculated by the delta delta-CT method, as previously described [46].

\section{Quantitative assessment of $\beta$-amyloid and $p$-tau deposits in brain tissues}

In order to quantitatively assess the $\beta$-amyloid and $p$-tau burden for further statistical analysis, we applied a method to quantify protein deposits. This method generates a numeric measurement that reflects the extent of $\beta$-amyloid and $p$-tau deposition. In the case of $p$-tau, not only neurofibrillary tangles but also neuritic plaques and neuropil threads are taken into account (Additional file 1: Figure S2). Sections of the hippocampus were examined after performing immunostaining with anti $\beta$-amyloid and anti-p-tau antibodies as described above in Human brain samples and neuropathological examination. Three pictures were obtained for each immunostained section by using an Olympus BX51 microscope at $\times 10$ magnification power. Focal deposit of $\beta$-amyloid, as described by Braak \& Braak (neuritic, immature, and compact plaque) [43], was manually determined and was further edited and analyzed with the ImageJ software (figure e-1). Then, $\beta$-amyloid plaque count, referred to as amyloid plaque score (APS) (Additional file 1: Figure S2) and total area of $\beta$-amyloid deposition were automatically measured by ImageJ and averaged for each section. Regarding p-tau deposit, representative pictures were analyzed with ImageJ software in order to obtain an average quantitative measure of the global p-tau deposit for each section (Additional file 1: Figure S2).

\section{Methylation in the TSS-associated region of TREM2 by bisulfite cloning sequencing}

Genomic DNA was isolated from hippocampal tissue by standard methods [47]. Next, 500 ng of genomic DNA was bisulfite converted using the EpiTect Bisulfite Kit (QIAGEN, Redwood City, CA, USA) according to the manufacturer's instructions. An amplicon spanning the TSS-associated region of TREM2 was amplified by PCR (Fig. 2a). Primer pair sequences were designed by MethPrimer [48] and are listed in Additional file 1: Table S1. Genomic map of the amplicon was drawn using the UCSC Genome Browser [49]. Next, PCR products were cloned using the TopoTA Cloning System (Invitrogen, Carlsbad, CA, USA), and a minimum of 20 independent clones were sequenced for each examined subject by Sanger sequencing [50].

\section{Determination of $5 \mathrm{hmC}$ in hippocampus samples}

We evaluate 5 -hydroxymethycytosine $(5 \mathrm{hmC})$ by performing 5-hydroxymethylated DNA immunoprecipitation combined with RT-qPCR (5hMeDIP-RT-qPCR). Genomic DNA (1500 ng) was sonicated to obtain fragments of 150-500 base pair length that were assessed by
$1 \%$ agarose gel electrophoretic analysis. Next, DNA containing $5 \mathrm{hmC}$ was enriched using the EpiQuik Hydroxymethylated DNA Immunoprecipitation (hMeDIP) Kit (Epigentek, Farmingdale, NY, USA), according to manufacturer's recommendations. Primer pair sequences are listed in Additional file 1: Table S1. Serial dilutions of samples were performed to determine amplification efficiency for each primer pair.

Real-time qPCR (RT-qPCR) reactions were performed in triplicate for each sample using Power SYBR ${ }^{\circ}$ Green PCR Master Mix (Invitrogen, Carlsbad, CA, USA) in a 7300 real-time PCR System (Applied Biosystems, Foster City, CA, USA). The PCR amplicon sizes range from 97 to $108 \mathrm{bp}$. All the primers used in RT-qPCR assays were tested for specificity as indicated above. The amplification reaction program included an initial step of $95{ }^{\circ} \mathrm{C}$ for $10 \mathrm{~min}$, followed by 40 cycles of $95^{\circ} \mathrm{C}$ for $15 \mathrm{~s}, 60^{\circ} \mathrm{C}$ for $1 \mathrm{~min}$, and a final melt curve analysis step. DNA control from the kit was included in the experiments. Fold enrichment was calculated as the ratio of amplification efficiency of each immunoprecipitated sample over the efficiency of non-immune IgG as of the following the formula: $2^{\mathrm{dCt}}$, where $\mathrm{dCt}=\mathrm{Ct}^{\mathrm{INPUT}}-\mathrm{Ct}^{\mathrm{hMeDIP}}$, and further normalized to the lowest detectable sample.

\section{Statistical data analysis}

Statistical analysis was performed with SPSS software version 21.0 (IBM, Inc., USA). Before performing differential analysis, we checked that all continuous variables showed a normal distribution, as per one-sample Kolgomorov-Smirnov test and the normal quantilquantil (QQ) plots. Summarized data from continuous variables were expressed as mean \pm standard deviation. Significance level for all comparisons was set at $p$ value $<0.05$. Statistical significance for intergroup differences was assessed by the Pearson's chi-square test for categorical variables and the independent sample $t$ test for continuous variables. After finding that variances were unequal by Levene's test, one-way analysis of variance (ANOVA) followed by Games-Howell post hoc analysis was used to analyze differences in the expression levels of TREM 2 mRNA between stage groups. A logistic regression model (ENTER method) was fit to assess the independent association of TREM2 mRNA levels with AD status, using gender and age as covariates. The Pearson product-moment correlation coefficient analysis was used to correlate TREM2 mRNA expression levels with neuropathological changes. Methylation ratio was calculated as the ratio of methylated CpGs over the total number of $\mathrm{CpGs}$ assessed for each CpG site. Gain in methylation was calculated as the subtraction of percentage of methylation between the $\mathrm{AD}$ group and control group. Although methylation is not completely independent for each CpG site within the same amplicon, a 
conservative approach to avoid false positive results were used by applying Bonferroni correction. Difference between two bisulfite sequence groups was evaluated with Mann-Whitney $U$ test. GraphPad Prism version 6.00 for Windows (GraphPad Software, La Jolla, CA, USA) was used to draw the graphs except for methylation figures that were drawn by QUMA software [51].

\section{Additional file}

Additional file 1: Contains supplemental Figure S1 (Both TREM2 splice variants showed increased mRNA expression in human hippocampus in Alzheimer's disease), Figure S2 (Beta-amyloid and tau protein measurement in hippocampal sections) and Figure $\mathbf{S 3}$ (A negative control upstream the TREM2 promoter was surveyed by bisulfite cloning sequencing) along with supplemental Table S1 (primers) and Table S2 (subjects characteristics). (PDF $656 \mathrm{~kb}$ )

\section{Abbreviations}

5hmC: 5-hydroxymethycytosine; 5hMeDIP: 5-hydroymethylated DNA immunoprecipitation; 5mC: 5-methylcytosine; AD: Alzheimer's disease; APS: amyloid plaque score; CpGs: cytosine guanine dinucleotides; GWAS: genome-wide association studies; p-tau: hyperphosphorylated tau; RT-qPCR: real-time qPCR; TREM2: triggering receptor expressed on myeloid cells 2.

\section{Competing interest}

The authors declare that they have no competing interests

\section{Authors' contributions}

NC contributed to drafting/revising the manuscript for content, analysis and interpretation of data, acquisition of data, and statistical analysis. JSR contributed to drafting/revising the manuscript for content, analysis and interpretation of data, and acquisition of data. MVZ contributed to drafting/ revising the manuscript for content, analysis and interpretation of data, and acquisition of data. MR performed experiments and was involved in interpretation of the data. RL contributed to acquisition of data. LP contributed to drafting/revising the manuscript for content and analysis and interpretation of data. CE contributed to drafting/revising the manuscript for content and acquisition of data. MM contributed to drafting/revising the manuscript for content, study concept and design, analysis and interpretation of data, acquisition of data, statistical analysis, study supervision, and obtaining funding. All authors read and approved the final manuscript.

\section{Acknowledgements}

This work was supported by the Spanish Government through a grant from the Institute of Health Carlos III (FIS PI13/02730), jointly funded by European Regional Development Fund (ERDF) and European Union, "A way of shaping Europe"; the Regional Basque Government through a grant from The Basque Foundation for Health Innovation and Research (BIOEF) (BIO12/ALZ/007), a grant from Fundación Caja-Navarra; and the Trans-Pyrenean Biomedical Research Network (REFBIO). NC was also supported by REFBIO funding. We want to kindly thank Teresa Tuñón M.D., Ph.D. (Department of Pathology, Complejo Hospitalario de Navarra, technical support), Federico García-Bragado M.D., Ph.D. (Department of Pathology, Complejo Hospitalario de Navarra, technical support), Iván Méndez M.D., (Department of Internal Medicine, Hospital García Orcoyen, technical editing), and Isabel Gil M.D. (Navarrabiomed BrainBank, technical support) for their help. Finally, we are very grateful to the patients and relatives that generously donor the brain tissue to the Navarrabiomed Brain Bank.

\section{Author details}

${ }^{1}$ Neuroepigenetics Laboratory, Navarrabiomed-Navarra Institute for Health Research (IdiSNA), c/ Irunlarrea, Pamplona, Navarra 31008, Spain. ${ }^{2}$ Department of Neurology, Complejo Hospitalario de Navarra, Pamplona, Navarra 31008, Spain. ${ }^{3}$ Department of Pathology, Complejo Hospitalario de
Navarra, Pamplona, Navarra 31008, Spain. ${ }^{4}$ Hospital Psicogeriátrico Josefina Arregui, Alsasua, Navarra 31800, Spain. ${ }^{5}$ Present address: Clínica San Miguel, Pamplona, Navarra 31006, Spain.

Received: 18 September 2015 Accepted: 22 March 2016

Published online: 05 April 2016

\section{References}

1. Hsieh CL, Koike M, Spusta SC, Niemi EC, Yenari M, Nakamura MC, et al. A role for TREM2 ligands in the phagocytosis of apoptotic neuronal cells by microglia. J Neurochem. 2009;109(4):1144-56. doi:10.1111/j.1471-4159.2009. 06042.x.

2. Stefano L, Racchetti G, Bianco F, Passini N, Gupta RS, Panina Bordignon P, et al. The surface-exposed chaperone, Hsp60, is an agonist of the microglial TREM2 receptor. J Neurochem. 2009;110(1):284-94. doi:10.1111/j.1471-4159. 2009.06130.x.

3. Takahashi K, Rochford CD, Neumann H. Clearance of apoptotic neurons without inflammation by microglial triggering receptor expressed on myeloid cells-2. J Exp Med. 2005;201(4):647-57. doi:10.1084/jem.20041611.

4. Rayaprolu S, Mullen B, Baker M, Lynch T, Finger E, Seeley WW, et al. TREM2 in neurodegeneration: evidence for association of the p.R47H variant with frontotemporal dementia and Parkinson's disease. Mol Neurodegener. 2013;8:19. doi:10.1186/1750-1326-8-19.

5. Cady J, Koval ED, Benitez BA, Zaidman C, Jockel-Balsarotti J, Allred P, et al. TREM2 variant p.R47H as a risk factor for sporadic amyotrophic lateral sclerosis. JAMA Neurol. 2014;71(4):449-53. doi:10.1001/jamaneurol.2013.6237.

6. Guerreiro R, Wojtas A, Bras J, Carrasquillo M, Rogaeva E, Majounie E, et al. TREM2 variants in Alzheimer's disease. N Engl J Med. 2013;368(2):117-27. doi:10.1056/NEJMoa1211851.

7. Jonsson T, Stefansson H, Steinberg S, Jonsdottir I, Jonsson PV, Snaedal J, et al. Variant of TREM2 associated with the risk of Alzheimer's disease. N Engl J Med. 2013;368(2):107-16. doi:10.1056/NEJMoa1211103.

8. Ulrich JD, Finn MB, Wang $Y$, Shen $A$, Mahan TE, Jiang $H$, et al. Altered microglial response to Abeta plaques in APPPS1-21 mice heterozygous for TREM2. Mol Neurodegener. 2014;9:20. doi:10.1186/1750-1326-9-20.

9. Lue LF, Schmitz C, Walker DG. What happens to microglial TREM2 in Alzheimer's disease: immunoregulatory turned into immunopathogenic? Neuroscience. 2014. doi:10.1016/j.neuroscience.2014.09.050.

10. Rohn TT. The triggering receptor expressed on myeloid cells 2: "TREM-ming" the inflammatory component associated with Alzheimer's disease. Oxid Med Cell Longev. 2013;2013:860959. doi:10.1155/2013/860959.

11. Kleinberger G, Yamanishi Y, Suarez-Calvet M, Czirr E, Lohmann E, Cuyvers E, et al. TREM2 mutations implicated in neurodegeneration impair cell surface transport and phagocytosis. Sci Transl Med. 2014;6(243):243ra86. doi:10. 1126/scitranslmed.3009093.

12. Abduljaleel Z, Al-Allaf FA, Khan W, Athar M, Shahzad N, Taher MM, et al. Evidence of trem2 variant associated with triple risk of Alzheimer's disease. PLoS One. 2014;9(3):e92648. doi:10.1371/journal.pone.0092648.

13. Zhao Y, Hill JM, Bhattacharjee S, Percy ME, Pogue Al, Lukiw WJ. Aluminuminduced amyloidogenesis and impairment in the clearance of amyloid peptides from the central nervous system in Alzheimer's disease. Front Neurol. 2014;5:167. doi:10.3389/fneur.2014.00167.

14. Jiang T, Tan L, Zhu XC, Zhang QQ, Cao L, Tan MS, et al. Upregulation of TREM2 ameliorates neuropathology and rescues spatial cognitive impairment in a transgenic mouse model of Alzheimer's disease. Neuropsychopharmacology. 2014;39(13):2949-62. doi:10.1038/npp.2014.164.

15. Wang Y, Cella M, Mallinson K, Ulrich JD, Young KL, Robinette ML, et al. TREM2 lipid sensing sustains the microglial response in an Alzheimer's disease model. Cell. 2015;160(6):1061-71. doi:10.1016/j.cell.2015.01.049.

16. Hickman SE, El Khoury J. TREM2 and the neuroimmunology of Alzheimer's disease. Biochem Pharmacol. 2014;88(4):495-8. doi:10.1016/j.bcp.2013.11.021.

17. Frank S, Burbach GJ, Bonin M, Walter M, Streit W, Bechmann I, et al. TREM2 is upregulated in amyloid plaque-associated microglia in aged APP23 transgenic mice. Glia. 2008;56(13):1438-47. doi:10.1002/glia.20710.

18. Forabosco P, Ramasamy A, Trabzuni D, Walker R, Smith C, Bras J, et al. Insights into TREM2 biology by network analysis of human brain gene expression data. Neurobiol Aging. 2013;34(12):2699-714. doi:10.1016/j. neurobiolaging.2013.05.001.

19. Martiskainen H, Viswanathan J, Nykanen NP, Kurki M, Helisalmi S, Natunen T, et al. Transcriptomics and mechanistic elucidation of Alzheimer's disease risk 
genes in the brain and in vitro models. Neurobiol Aging. 2014. doi:10.1016/j. neurobiolaging.2014.09.003.

20. Strobel $\mathrm{S}$, Grunblatt E, Riederer P, Heinsen H, Arzberger T, Al-Sarraj $S$ et al. Changes in the expression of genes related to neuroinflammation over the course of sporadic Alzheimer's disease progression: CX3CL1, TREM2, and PPARgamma. J Neural Transm. 2015. doi:10.1007/s00702-015-1369-5.

21. Lue LF, Schmitz CT, Serrano G, Sue LI, Beach TG, Walker DG. TREM2 protein expression changes correlate with Alzheimer's disease neurodegenerative pathologies in post-mortem temporal cortices. Brain Pathol (Zurich, Switzerland). 2015;25(4):469-80. doi:10.1111/bpa.12190.

22. Zhao Y, Bhattacharjee S, Jones BM, Dua P, Alexandrov PN, Hill JM, et al. Regulation of TREM2 expression by an NF-small ka, CyrillicB-sensitive miRNA-34a. Neuroreport. 2013;24(6):318-23. doi:10.1097/WNR. 0b013e32835fb6b0.

23. Schneider CA, Rasband WS, Eliceiri KW. NIH Image to ImageJ: 25 years of image analysis. Nat Methods. 2012;9(7):671-5.

24. Mellen M, Ayata P, Dewell S, Kriaucionis S, Heintz N. MeCP2 binds to $5 \mathrm{hmC}$ enriched within active genes and accessible chromatin in the nervous system. Cell. 2012;151(7):1417-30.

25. Colquitt BM, Allen WE, Barnea G, Lomvardas S. Alteration of genic 5hydroxymethylcytosine patterning in olfactory neurons correlates with changes in gene expression and cell identity. Proc Natl Acad Sci U S A. 2013;110(36): 14682-7. doi:10.1073/pnas.1302759110.

26. Korvatska O, Leverenz JB, Jayadev S, McMillan P, Kurtz I, Guo X et al. R47H variant of TREM2 associated with Alzheimer disease in a large late-onset family: clinical, genetic, and neuropathological study. JAMA Neurol. 2015. doi:10.1001/jamaneurol.2015.0979.

27. Pottier C, Wallon D, Rousseau S, Rovelet-Lecrux A, Richard AC, Rollin-Sillaire A, et al. TREM2 R47H variant as a risk factor for early-onset Alzheimer's disease. J Alzheimers Dis. 2013;35(1):45-9. doi:10.3233/jad-122311.

28. Tang Y, Li T, Li J, Yang J, Liu H, Zhang XJ, et al. Jmjd3 is essential for the epigenetic modulation of microglia phenotypes in the immune pathogenesis of Parkinson's disease. Cell Death Differ. 2014;21 (3):369-80

29. Weber M, Hellmann I, Stadler MB, Ramos L, Paabo S, Rebhan M, et al. Distribution, silencing potential and evolutionary impact of promoter DNA methylation in the human genome. Nat Genet. 2007;39(4):457-66.

30. Villar-Menendez I, Blanch M, Tyebji S, Pereira-Veiga T, Albasanz JL, Martin $\mathrm{M}$, et al. Increased 5-methylcytosine and decreased 5hydroxymethylcytosine levels are associated with reduced striatal A2AR levels in Huntington's disease. Neuromolecular Med. 2013;15(2):295-309. doi:10.1007/s12017-013-8219-0.

31. Selamat SA, Chung BS, Girard L, Zhang W, Zhang Y, Campan M, et al. Genome-scale analysis of DNA methylation in lung adenocarcinoma and integration with mRNA expression. Genome Res. 2012;22(7):1197-211. doi:10.1101/gr.132662.111.

32. Munzel M, Globisch D, Bruckl T, Wagner M, Welzmiller V, Michalakis S, et al. Quantification of the sixth DNA base hydroxymethylcytosine in the brain. Angewandte Chemie. 2010;49(31):5375-7. doi:10.1002/anie.201002033.

33. Kriaucionis $\mathrm{S}$, Heintz $\mathrm{N}$. The nuclear DNA base 5 -hydroxymethylcytosine is present in Purkinje neurons and the brain. Science. 2009;324(5929):929-30. doi:10.1126/science.1169786.

34. Szulwach KE, Li X, Li Y, Song CX, Wu H, Dai Q, et al. 5-hmC-mediated epigenetic dynamics during postnatal neurodevelopment and aging. Nat Neurosci. 2011;14(12):1607-16. doi:10.1038/nn.2959.

35. Chouliaras L, van den Hove DL, Kenis G, Keitel S, Hof PR, van Os J, et al. Agerelated increase in levels of 5-hydroxymethylcytosine in mouse hippocampus is prevented by caloric restriction. Curr Alzheimer Res. 2012;9(5):536-44.

36. Chen H, Dzitoyeva S, Manev H. Effect of aging on 5-hydroxymethylcytosine in the mouse hippocampus. Restor Neurol Neurosci. 2012;30(3):237-45. doi:10.3233/RNN-2012-110223.

37. Coppieters N, Dieriks BV, Lill C, Faull RL, Curtis MA, Dragunow M. Global changes in DNA methylation and hydroxymethylation in Alzheimer's disease human brain. Neurobiol Aging. 2014;35(6):1334-44. doi:10.1016/j. neurobiolaging.2013.11.031.

38. Wu H, D'Alessio AC, Ito S, Wang Z, Cui K, Zhao K, et al. Genome-wide analysis of 5-hydroxymethylcytosine distribution reveals its dual function in transcriptional regulation in mouse embryonic stem cells. Genes Dev. 2011;25(7):679-84. doi:10.1101/gad.2036011.

39. Irwin RE, Thakur A, O' Neill KM, Walsh CP. 5-Hydroxymethylation marks a class of neuronal gene regulated by intragenic methylcytosine levels. Genomics. 2014;104(5):383-92.
40. Bell JE, Alafuzoff I, Al-Sarraj S, Arzberger T, Bogdanovic N, Budka H, et al. Management of a twenty-first century brain bank: experience in the BrainNet Europe consortium. Acta Neuropathol. 2008;115(5):497-507. doi:10.1007/s00401-008-0360-8.

41. Braak H, Alafuzoff I, Arzberger T, Kretzschmar H, Del Tredici K. Staging of Alzheimer disease-associated neurofibrillary pathology using paraffin sections and immunocytochemistry. Acta Neuropathol. 2006;112(4):389-404. doi:10.1007/s00401-006-0127-z.

42. McKhann GM, Knopman DS, Chertkow H, Hyman BT, Jack Jr CR, Kawas CH, et al. The diagnosis of dementia due to Alzheimer's disease: recommendations from the National Institute on Aging-Alzheimer's Association workgroups on diagnostic guidelines for Alzheimer's disease. Alzheimers Dement. 2011;7(3):263-9. doi:10.1016/j.jalz.2011.03.005.

43. Braak H, Braak E. Neuropathological stageing of Alzheimer-related changes. Acta Neuropathol. 1991;82(4):239-59.

44. Lue LF, Schmitz CT, Serrano G, Sue LI, Beach TG, Walker DG. TREM2 protein expression changes correlate with Alzheimer's disease neurodegenerative pathologies in post-mortem temporal cortices. Brain Pathol (Zurich, Switzerland). 2014; doi:10.1111/bpa.12190.

45. Vandesompele J, De Preter K, Pattyn F, Poppe B, Van Roy N, De Paepe A, et al. Accurate normalization of real-time quantitative RT-PCR data by geometric averaging of multiple internal control genes. Genome Biol. 2002;3(7):RESEARCH0034.

46. Livak KJ, Schmittgen TD. Analysis of relative gene expression data using real-time quantitative PCR and the 2(-Delta Delta C(T)) method. Methods (San Diego, Calif). 2001;25(4):402-8. doi:10.1006/meth.2001.1262.

47. Miller SA, Dykes DD, Polesky HF. A simple salting out procedure for extracting DNA from human nucleated cells. Nucleic Acids Res. 1988;16(3):1215.

48. Li LC, Dahiya R. MethPrimer: designing primers for methylation PCRs. Bioinformatics. 2002;18(11):1427-31.

49. Kent WJ, Sugnet CW, Furey TS, Roskin KM, Pringle TH, Zahler AM, et al. The human genome browser at UCSC. Genome Res. 2002;12(6):996-1006. doi:10.1101/gr.229102. Article published online before print in May 2002.

50. Sanger F, Nicklen S, Coulson AR. DNA sequencing with chain-terminating inhibitors. Proc Natl Acad Sci U S A. 1977;74(12):5463-7.

51. Kumaki Y, Oda M, Okano M. QUMA: quantification tool for methylation analysis. Nucleic Acids Res. 2008;36(Web Server issue):W170-5. doi:10.1093/ nar/gkn294

\section{Submit your next manuscript to BioMed Central and we will help you at every step:}

- We accept pre-submission inquiries

- Our selector tool helps you to find the most relevant journal

- We provide round the clock customer support

- Convenient online submission

- Thorough peer review

- Inclusion in PubMed and all major indexing services

- Maximum visibility for your research

Submit your manuscript at www.biomedcentral.com/submit
( Biomed Central 